\title{
Left ventricular remodelling and brain natriuretic peptide after first myocardial infarction
}

\author{
J G Crilley, M Farrer
}

\begin{abstract}
Objective-To investigate the relation between brain natriuretic peptide (BNP) concentrations and left ventricular remodelling characteristics after acute myocardial infarction.

Design-Consecutive sample prospective cohort study.

Setting-District general hospital coronary care unit in the north of England.

Patients-133 initial survivors of a first myocardial infarction who received thrombolytic treatment.

Interventions-Patients had transthoracic echocardiography and BNP concentrations measured at three to seven days (early) and two months (late).

Main outcome measures-Wall motion index $\leqslant 1.2$, end systolic left ventricular volume index and mortality at one year.

Results-Patients with an early wall motion index of $\leqslant 1.2$ had higher early and late BNP concentrations (early BNP mean (SEM) $629(76.2) \mathrm{pg} / \mathrm{ml} v 334(20.8) \mathrm{pg} / \mathrm{ml}, \mathrm{p}=0.001$ and late BNP $584(79.5) \mathrm{pg} / \mathrm{ml} v 343(25.0) \mathrm{pg} / \mathrm{ml}, \mathrm{p}=0.001)$. Patients with an increase in end systolic left ventricular volume index of $>10 \%$ also had higher early and late BNP concentrations (early BNP $p=0.034$ and late BNP $p=0.001)$. Early BNP was significantly associated with one year mortality $(\mathrm{p}=0.003)$.

Conclusions-Higher BNP concentrations early after first myocardial infarction are associated with adverse left ventricular remodelling characteristics. This may help explain why BNP is such a strong predictor of outcome after myocardial infarction.

(Heart 2001;86:638-642)
\end{abstract}

Keywords: brain natriuretic peptide; wall motion index; left ventricular volume index; myocardial infarction

Brain natriuretic peptide (BNP) is a cardiac hormone belonging to the natriuretic peptide family. Its functions are diverse and include diuresis, natriuresis, vasodilatation, and inhibition of the renin-angiotensin system. BNP was first isolated from porcine brain ${ }^{1}$ and later found in human heart. $^{23}$ Although it has significant sequence homology with atrial natriuretic peptide, it is secreted primarily from and synthesised in the left ventricle in response to increased myocardial stretch. ${ }^{4}$ It is now an accepted marker of left ventricular (LV) systolic dysfunction and several studies have explored its ability to identify asymptomatic patients with LV systolic dysfunction who may benefit from angiotensin converting enzyme inhibitors. ${ }^{5-8}$ Notably increased BNP concentrations have also been reported after acute myocardial infarction $(\mathrm{MI})^{9}$ and a high BNP early after MI appears to be a strong predictor of longer term mortality. ${ }^{10-14}$ The mechanism behind this remains to be fully explored. Adverse LV remodelling is also known to be a strong predictor of outcome after MI. ${ }^{15}$ Three small recently published studies ${ }^{16-18}$ have explored this mechanism in selected groups of patients. We have investigated, using echocardiography, in an unselected cohort of MI patients who received current standard hospital care whether adverse LV remodelling can contribute to understanding the mechanism by which $\mathrm{BNP}$ is such a strong predictor of outcome after MI.

\section{Methods}

PATIENTS

The study group comprised 285 patients who were admitted to the coronary care unit at Sunderland Royal Hospital between December 1996 and October 1997 with a presumed diagnosis of MI and who received thrombolysis. One hundred and thirty three patients met the entry criteria of first MI and survival to three days. Patients were excluded if they had a second or subsequent MI (61); the diagnostic criteria for MI (15) were not met; they died after less than three days (16); they experienced a reinfarction or unstable angina within less than three days (13); there was a poor echocardiography window (8); the patient was unable to or refused to give consent (7); the sample was unsuitable for BNP analysis (9); the patient was prematurely discharged (23). MI was diagnosed if two of three of the following criteria were met: chest pain lasting longer than 30 minutes, $>1 \mathrm{~mm}$ ST elevation in two limb leads or $>2 \mathrm{~mm}$ ST elevation in two chest leads, or a rise in creatine kinase to twice the upper limit of normal. Thrombolytic treatment was given if patients presented within 12 hours of the onset of chest pain. There was no age limit and conventional contraindications to thrombolysis were observed. Streptokinase (1.5 MU over 60 minutes) was given unless the patient was hypotensive on presentation (systolic blood pressure $<90 \mathrm{~mm} \mathrm{Hg}$ ) or if streptokinase intolerance or allergy was possible. Intravenous front loaded tissue type plasminogen activator was given in these circumstances. 
Twelve lead ECGs were recorded at 0, 60, 90, and 180 minutes after thrombolysis and were used for later analysis of reperfusion. Patients underwent transthoracic echocardiography and had blood samples taken for BNP analysis at three to seven days, defined as the early time point, and at two months, defined as the late time point.

\section{OUTCOMES}

Patients were followed up at one year by postal questionnaire. All patients who were alive returned the questionnaire. Causes of death were confirmed by obtaining the death certificate from the Office of National Statistics, London, UK, and were classified as cardiac or non-cardiac.

ECG ANALYSIS

ECGs were analysed for evidence of reperfusion in a manner identical to that described previously. ${ }^{19}$ Briefly, the height of the ST segment in the lead of maximum elevation on the baseline ECG was measured by caliper at $80 \mathrm{~ms}$ after the J point. ECGs could not be evaluated in $11 \%$ of patients because of conduction abnormalities or pure posterior infarction. A 50\% fall in ST segment height was taken as evidence of reperfusion.

ECHOCARDIOGRAPHY

All scans were performed on a Sonos 1500 (Hewlett Packard, Bracknall, UK). The majority of early and late scans were performed by the same operator. Intraobserver variability was $5 \%$. The following parameters were measured and averaged over three cardiac cycles: end systolic volume (ESV), end diastolic volume (EDV) (using modified Simpson's rule), ejection fraction (calculated from LV volumes), and wall motion index (WMI) (nine segment model). ESV and EDV were corrected for body surface area to give the ESV index (ESVi) and EDV index (EDVi). The percentage change in ESVi and EDVi between the early and late studies was calculated. A WMI cut off of $\leqslant 1.2$ was used to indicate LV dysfunction.

BNP ANALYSIS

Venous samples were centrifuged promptly and stored at $-80^{\circ} \mathrm{C}$ until analysis. All samples were analysed as a single batch. BNP was measured using a direct (non-extracted) radioimmunoassay (Peninsula Laboratories Inc, Belmont, California, USA). This was a two day staggered incubation assay using an antibody specific for $\mathrm{BNP}$ (1-32) and a radiolabelled BNP tracer $\left({ }^{125} \mathrm{I}-\mathrm{BNP}\right)$.

STATISTICAL ANALYSIS

Results are presented as mean (SD) and BNP data as mean (SEM) with a significance level of $<0.05$ being accepted. Statistical analysis was facilitated by the use of the SPSS (version 10) software package (SPSS Inc, Chicago, Illinois, USA). An unpaired $t$ test or one way analysis of variance (ANOVA) was used to explore the influence of categorical variables on early BNP and late BNP. Receiver operating characteristic curves were generated using SPSS for early
Table 1 Baseline characteristics of patient cohort

\begin{tabular}{|c|c|c|}
\hline & Mean (SD) & $n(\%)$ \\
\hline Age (years) & $62(10.7)$ & \\
\hline Male & & $93(70)$ \\
\hline Diabetes & & $12(9)$ \\
\hline Hypertension & & $29(22)$ \\
\hline Smoker & & $69(52)$ \\
\hline Cholesterol (mmol/1) & $6.0(1.25)$ & \\
\hline Creatine $(\mathrm{mmol} / \mathrm{l})$ & $106(19.0)$ & \\
\hline Anterior myocardial infarction & & $56(42)$ \\
\hline $\begin{array}{l}\text { Tissue type plasminogen } \\
\text { activator given }\end{array}$ & & $8(6)$ \\
\hline Angiotensin converting enzyme & & $61(48)$ \\
\hline$\beta$ blocker & & $89(70)$ \\
\hline Aspirin & & $117(91)$ \\
\hline Cholesterol lowering agent & & $37(29)$ \\
\hline Early ESVi $\left(\mathrm{ml} / \mathrm{m}^{2}\right)$ & $26(9.7)$ & \\
\hline Early EDVi $\left(\mathrm{ml} / \mathrm{m}^{2}\right)$ & $45(12.9)$ & \\
\hline Early ejection fraction (\%) & $44(9.7)$ & \\
\hline Early WMI & $1.5(0.38)$ & \\
\hline Late ESVi $\left(\mathrm{ml} / \mathrm{m}^{2}\right)$ & $27(10.8)$ & \\
\hline Late $\mathrm{EDVi}\left(\mathrm{ml} / \mathrm{m}^{2}\right)$ & $46(14.1)$ & \\
\hline Late ejection fraction (\%) & $43(9.2)$ & \\
\hline Late WMI & $1.6(0.38)$ & \\
\hline
\end{tabular}

EDVi, end diastolic volume index; ESVi, end systolic volume index; WMI, wall motion index

$\mathrm{BNP}$ and late BNP against the dichotomous variables of early WMI $\leqslant 1.2$ and late WMI $\leqslant 1.2$. We used multiple regression with a stepwise procedure for variable selection to model early BNP and late BNP as a function of the covariables that on univariate analysis gave significant results.

\section{Results}

BASELINE CHARACTERISTICS

Of the 133 patients recruited to the study, five died before the two month assessment, leaving 128 subjects for whom there were two sets of echocardiography and BNP data. Table 1 summarises the characteristics of the patients. Causes of the five deaths between the early and late assessments were all cardiac. There were four further deaths (three cardiac) by one year (overall mortality $7 \%$ ).

BNP AND ONE YEAR MORTALITY

Early BNP was strongly associated with cardiac mortality by one year (cardiac death: 675 (134.5) $\mathrm{pg} / \mathrm{ml} v$ alive at one year: 365 (21.8) $\mathrm{pg} / \mathrm{ml} ; \mathrm{p}<0.003)$. Late BNP was more weakly associated with mortality at one year (cardiac death: 737 (293) pg/ml $v$ alive at one year: 368 (24.8) $\mathrm{pg} / \mathrm{ml} ; \mathrm{p}=0.06)$. Only one death occurred among patients whose early $\mathrm{BNP}$ was below the group median of $301 \mathrm{pg} /$ $\mathrm{ml}$ (fig 1).

BNP AND LV REMODELLING CHARACTERISTICS

$B N P$ and WMI

There was a strong association between an early WMI of $\leqslant 1.2$ and early BNP and late BNP (fig 2). Patients with a low WMI at this early time point had higher early and maintained higher late BNP concentrations. Late WMI was closely related to late BNP (late WMI $\leqslant 1.2: 558(95.7) \mathrm{pg} / \mathrm{ml} v$ late WMI > 1.2: 349 (24.7) pg/ml; $\mathrm{p}=0.049)$ but not to early BNP. A receiver operating characteristic curve showed that the optimal value of early BNP for predicting an early WMI of $>1.2$ is $456 \mathrm{pg} / \mathrm{ml}$ (sensitivity $73 \%$, specificity $78 \%$ ) (fig 3). 


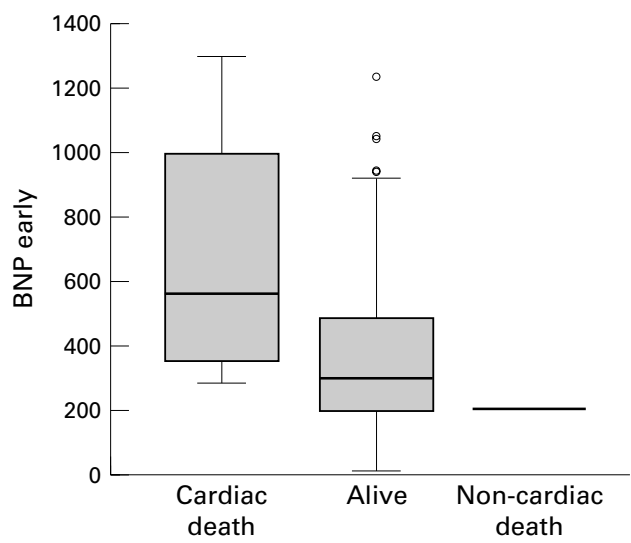

Figure 1 Box plot showing median, interquartile range (grey box), sample range (error bars) and outliers (open circles) for brain natriuretic peptide (BNP) measured at three to seven days (early) versus cardiac death, alive at one year, and non-cardiac death.

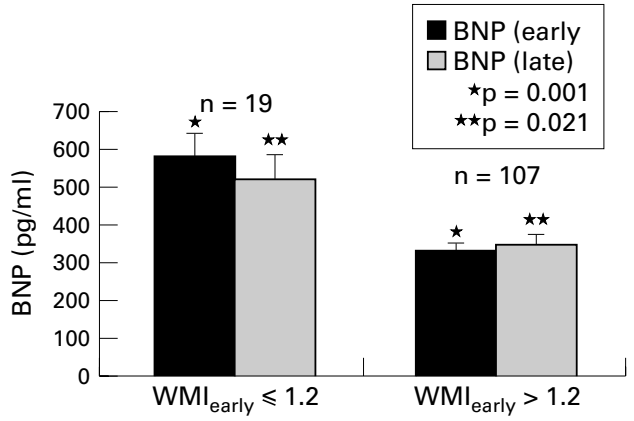

Figure 2 Bar chart showing mean early BNP (black bars) and late BNP (grey bars) versus early wall motion index $(W M I) \leqslant 1.2$ and early $W M I>1.2$. $n$, number in each group.

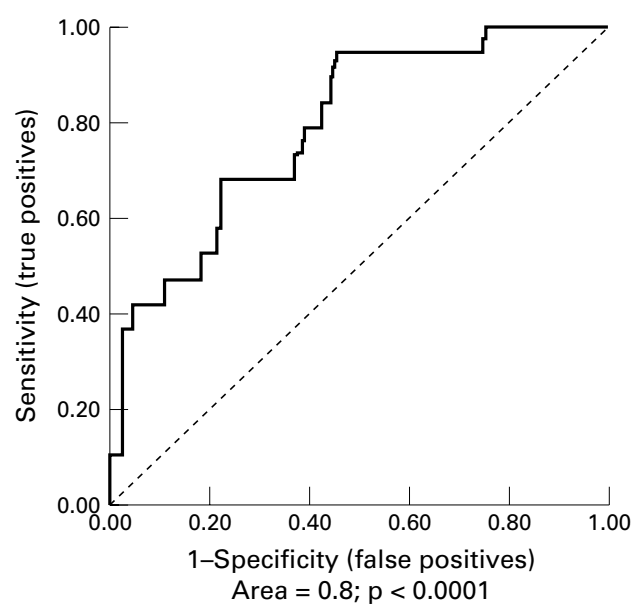

Figure 3 Receiver operating characteristic curve for early $B N P$ versus early $W M I \leqslant 1.2$.

$B N P$ and $L V$ volume indices

There was a strong association in the percentage change in ESVi between the early and late studies and between early BNP and late BNP. Those patients with a change in ESVi of $>10 \%$ had higher early and late BNP concentrations (fig 4). The association was stronger for the late than for the early BNP concentration. There was a trend towards a lower late $\mathrm{BNP}$ in patients with a change in EDVi of $<10 \%$ (298 (31.2) $\mathrm{pg} / \mathrm{ml} v 412(47) \mathrm{pg} / \mathrm{ml}$ ) but this was not significant. There was no relation between

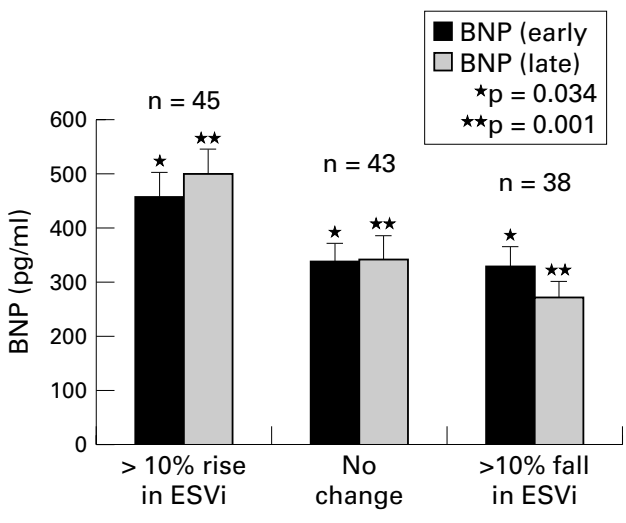

Figure 4 Bar chart showing mean early BNP (black bars) and late BNP (grey bars) versus $>10 \%$ rise in end systolic volume index (ESVi), no change, and $>10 \%$ fall in ESVi.

early BNP and change in EDVi nor between $\mathrm{BNP}$ and change in ejection fraction.

BNP AND REPERFUSION

There was no association between failure to reperfuse by 60 or 90 minutes (according to ECG criteria as previously described) and early $\mathrm{BNP}$ or late BNP. However, failure to reperfuse by 180 minutes was associated with higher early but not late BNP concentrations (489 (48.4) $\mathrm{pg} / \mathrm{ml} v 331$ (26.3) $\mathrm{pg} / \mathrm{ml} ; \mathrm{p}<0.003)$.

BNP AND SITE OF INFARCT

Early BNP was significantly higher in patients with an anterior MI than in those with inferior or lateral infarcts (anterior MI: 453 (37.8) pg/ $\mathrm{ml}$; inferior MI: 336 (28.1) pg/ml; $\mathrm{p}=0.026)$

BNP AND OTHER VARIABLES

There was no association between early BNP or late BNP and ejection fraction (early or late), demographic variables, risk factors for coronary disease, or drug treatment given for secondary prevention.

MULTIPLE REGRESSION MODEL

The results of the multiple linear regression model showed that the strongest association with early BNP was an early WMI of $\leqslant 1.2$ $(\mathrm{p}<0.001)$, closely followed by ESVi dilatation of $>10 \%(p<0.028)$. After excluding the influence of these variables, site of infarct, time to reperfusion, and status at 12 months did not show any independent association with early BNP. Similarly the variables with the strongest association with late BNP were those associated with adverse remodelling characteristics: ESVi dilatation $>10 \%(\mathrm{p}<0.001)$ and early WMI $\leqslant 1.2(\mathrm{p}<0.006)$.

\section{Discussion}

The data from this study show that, as in previous studies, BNP measured in the first few days after MI is a strong predictor of mortality at one year. We have also shown that higher BNP concentrations at both early and late time points are closely linked to WMI and progressive LV systolic dilatation. The multiple regression model showed that once the influence of these remodelling variables had been excluded, mortality, time to reperfusion, and 
site of infarct were no longer independently associated with BNP concentrations. This suggests that the ability of BNP early after acute MI to predict mortality relates to higher BNP concentrations being associated with adverse remodelling characteristics. However, the number of deaths was too small to directly link BNP concentrations, remodelling characteristics, and mortality. The study of White and colleagues ${ }^{15}$ was one of the first to highlight that the most important determinant of outcome after MI is an increased ESV rather than ejection fraction. Interventions that have improved outcome after MI have, in many cases, exerted their effects through beneficial alterations in the remodelling process. ${ }^{20-21}$ However, there are still significant numbers of patients who have an unfavourable outcome after MI, and a useful means of identifying patients who would be targets for more invasive and aggressive interventions would be helpful.

BNP AND LV REMODELLING

Three small studies ${ }^{16-18}$ in 30,33 , and 21 patients, respectively, had findings similar to ours. In the first two, LV angiography was used to measure $\mathrm{LV}$ function, and associations were found between higher BNP concentrations and changes in ESVi, EDVi, and ejection fraction. In the latter study electron beam computed tomography was used and similar findings were obtained. However, all studies recruited highly selected patients and none reported any longer term mortality data. We did not find such striking associations between BNP and change in EDVi, nor any association between BNP concentrations and change in ejection fraction. Several studies have shown that ejection fraction is particularly difficult to measure accurately and reproducibly in the immediate postinfarction period. $^{22}$ We therefore used a WMI of $\leqslant 1.2$ (nine segment model, as used in the TRACE (trandolapril cardiac evaluation study ${ }^{22}$ ) as a surrogate for reduced LV ejection fraction and found it to be a more reliable marker of $\mathrm{LV}$ dysfunction.

MECHANISM OF BNP RELEASE

BNP appears to be released in a biphasic pattern after acute MI. Most patients have high BNP concentrations in the first 24 hours after MI. ${ }^{9}$ Concentrations then fall (but not as far as preinfarction concentrations) before rising again over the next three to seven days in some patients. These patients may maintain increased concentrations of BNP for some weeks after the acute event. Although it would be tempting to conclude that the initial rise was caused by direct release from necrotic areas of infarcted myocardium, experimental studies have shown that there is increased BNP mRNA expression in the surviving myocytes in the peri-infarct areas (possibly mediated through augmented transforming growth factor $\beta$ and basic fibroblastic growth factor expression) and that this occurs soon after the acute event. ${ }^{4}$ The stimulus for this appears to be increased wall stress directly related to the infarction event. ${ }^{23}$ As time progresses increased ventricular loading conditions stimulate further BNP release. The systemic effects of BNP, including natriuresis and vasodilatation, may act as a protective mechanism to limit further infarct expansion.

\section{STUDY LIMITATIONS}

We chose to focus our study on patients receiving current secondary care after MI. We achieved a high rate of secondary prevention uptake, which was maintained at least to one year after the MI. The BNP concentrations are higher than in some previous studies of BNP after MI although the absolute range of values is not dissimilar. There are two possible explanations for this. Firstly, our patients were unselected and therefore may have been more unwell than cohorts of patients in these smaller studies and have more extensive coronary disease. Previous data from our unit (unpublished) has shown that more patients than expected have extensive inferior infarcts involving the right ventricle and that a higher percentage have triple vessel disease seen on angiography when it has been performed for clinical indications. Particularly high concentrations of BNP were reported in a study that specifically investigated patients with right ventricular dysfunction secondary to pulmonary hypertension. $^{24}$ The mean ejection fraction found in our study was also slightly lower than in other studies, ${ }^{16-18}$ supporting this conclusion, but was not dissimilar to that which we have previously reported. ${ }^{19}$ A second explanation could be a systematic error in the method of sampling or processing but this would not alter the interpretation of our data. The nonextracted/direct assay method for BNP generally results in higher measured BNP concentrations than the extracted method. ${ }^{716}$

\section{CONCLUSIONS}

We have found that BNP early after MI is an important predictor of outcome after first MI. The mechanism of this appears to be related to adverse remodelling characteristics in the first two months after the event.

We are grateful to Sister Carole Nelson and staff of the Coronary Care Unit, Ms Helen Murray of the Clinical Audit Department, and Drs Kishore Shetty and Richard Graham, all of Sunderland Royal Hospital, Sunderland, UK. We also thank Dr Salim Fredericks and Professor David Holt, Analytical Unit, St George's Hospital, London, UK, who performed the BNP assay.

1 Sudoh T, Kangawa $\mathrm{K}$, Minamino N, et al. A new natriuretic peptide in porcine brain. Nature 1988;332:78-81.

2 Mukoyama M, Nakao K, Hosada K, et al. Brain natriuretic peptide as a novel cardiac hormone in humans. $\mathcal{F}$ Clin Invest 1991;87:1402-12.

3 Yasue $\mathrm{H}$, Yoshimura $\mathrm{M}$, Sumida $\mathrm{H}$, et al. Localisation and mechanism of secretion of B-type natriuretic peptide in comparison with those of A-type natriuretic peptide in normal subjects and patients with heart failure. Circulation mal subjects and

4 Hama N, Itoh H, Shirakami G, et al. Rapid ventricular induction of brain natriuretic peptide gene expression in experimental acute myocardial infarction. Circulation 1995; 92:1558-64

5 Davidson NC, Abdelwahab NA, Hanson JK, et al. Comparison of atrial natriuretic peptide, B-type natriuretic peptide and $\mathrm{N}$-terminal proatrial natriuretic peptide as indicators of left ventricular systolic dysfunction. $A m \mathcal{F}$ Cardiol 1996;77:828-31.

6 McClure SJ, Caruana L, Davie AP. Cohort study of plasma natriuretic peptides for identifying left ventricular systolic dysfunction in primary care. BMF 1998;317:516-9.

7 Motwani JG, McAlpine H, Kennedy N, et al. Plasma brain natriuretic peptide as an indicator for angiotensinconverting enzyme inhibition after myocardial infarction. converting enzyme inhibition

8 McDonagh TA, Robb SD, Murdoch DR, et al. Biochemical detection of left ventricular systolic dysfunction. Lancet 1998;351:9-13 
9 Morita E, Yasue $\mathrm{H}$, Yoshimura $\mathrm{M}$, et al. Increased plasma levels of brain natriuretic peptide in patients with acute levels of brain natriuretic peptide in patients with
myocardial infarction. Circulation 1993;88:82-91.

10 Richards AM, Nicholls MG, Yandle TG, et al. Neuroendocrine prediction of left ventricular function and heart failure after acute myocardial infarction. Heart 1999;81:114-201.

11 Omland T, Aakvaag A, Bonarjee V, et al. Plasma brain natriuretic peptide as an indicator of left ventricular systolic function and long-term survival after acute myocardial infarction. Circulation 1996;93:1963-9.

12 Darbar D, Davidson NC, Gillespie N, et al. Diagnostic value of B-type natriuretic peptide concentrations in patients with acute myocardial infarction. Am $\mathcal{F}$ Cardiol 1996;78:284-7.

13 Arakawa N, Nakamura M, Aoki H, et al. Plasma brain natriuretic peptide concentrations predict survival after acute myocardial infarction. 7 Am Coll Cardiol 1996;27:1656-61.

14 Byrne J, Murdoch DR, Morton JJ, et al Natriuretic peptides and long-term survival after myocardial infarction [ab-

15 White HD, Norris RM, Brown MA, et al. Left ventricular endsystolic volume as the major determinant of survival after recovery from myocardial infarction. Circulation 1987;76:44-51.

16 Nagaya N, Nishikimi T, Goto Y, et al. Plasma brain natriuNagaya N, Nishikimi T, Goto Y, et al. Plasma brain natriu-
retic peptide is a biochemical marker for the prediction of retic peptide is a biochemical marker for the prediction of
progressive ventricular remodeling after acute myocardial progressive ventricular remodeling after

17 Yoshitomi Y, Nishikimi T, Kojima S. Plasma natriuretic peptides as indicators of left ventricular remodelling after MI. Int f Cardiol 1998;64:153-60.
18 Nagaya N, Goto Y, Nishikimi T, et al. Sustained elevation of plasma brain natriuretic peptide levels associated with progressive ventricular remodelling after acute myocardial infgressive ventricular remodelling a

19 Purcell IF, Newall N, Farrer M. Change in ST segment elevation 60 minutes after thrombolytic initiation predicts clinical outcome as accurately as later electrocardiographic changes. Heart 1997;78:465-71.

20 Marino P, Zanolla L, Zardini P, et al. Effect of streptokinase on left ventricular modelling and function after myocardial infarction: The GISSI (Gruppo Italiano per lo studio della Streptochinaisi nell'Infarcto Myocardio) trial. f Am Coll Cardiol 1989;14:1149-58.

21 Sharpe N, Smith H, Murphy J, et al. Treatment of patients with symptomless left ventricular dysfunction after myocardial infarction. Lancet 1988;i:255-9.

22 Kober L, Torp-Pedersen C, Carlsen J. An echocardiographic method for selecting high risk patients shortly after acute myocardial infarction for inclusion in multi-centre studies (as used in the TRACE study). Trandolapril cardiac evaluation. Eur Heart $\mathcal{F}$ 1994;15:1616-20.

23 Sumida $\mathrm{H}$, Yasue $\mathrm{H}$, Yoshimura $\mathrm{M}$, et al. Comparison of secretion pattern between A-type and B-type natriuretic pecretion pattern between A-type and B-type natriuretic peptides in patients with old

24 Nagaya N, Nishikimi T, Okano Y, et al. Plasma brain natriuretic peptide increase in proportion to the extent of right ventricular dysfunction in pulmonary hypertension. f $\mathrm{Am}$ Coll Cardiol 1998;31:202-8.

\section{IMAGES IN CARDIOLOGY}

\section{Successful treatment of atrial tachycardia-induced cardiomyopathy with pilsicainide}
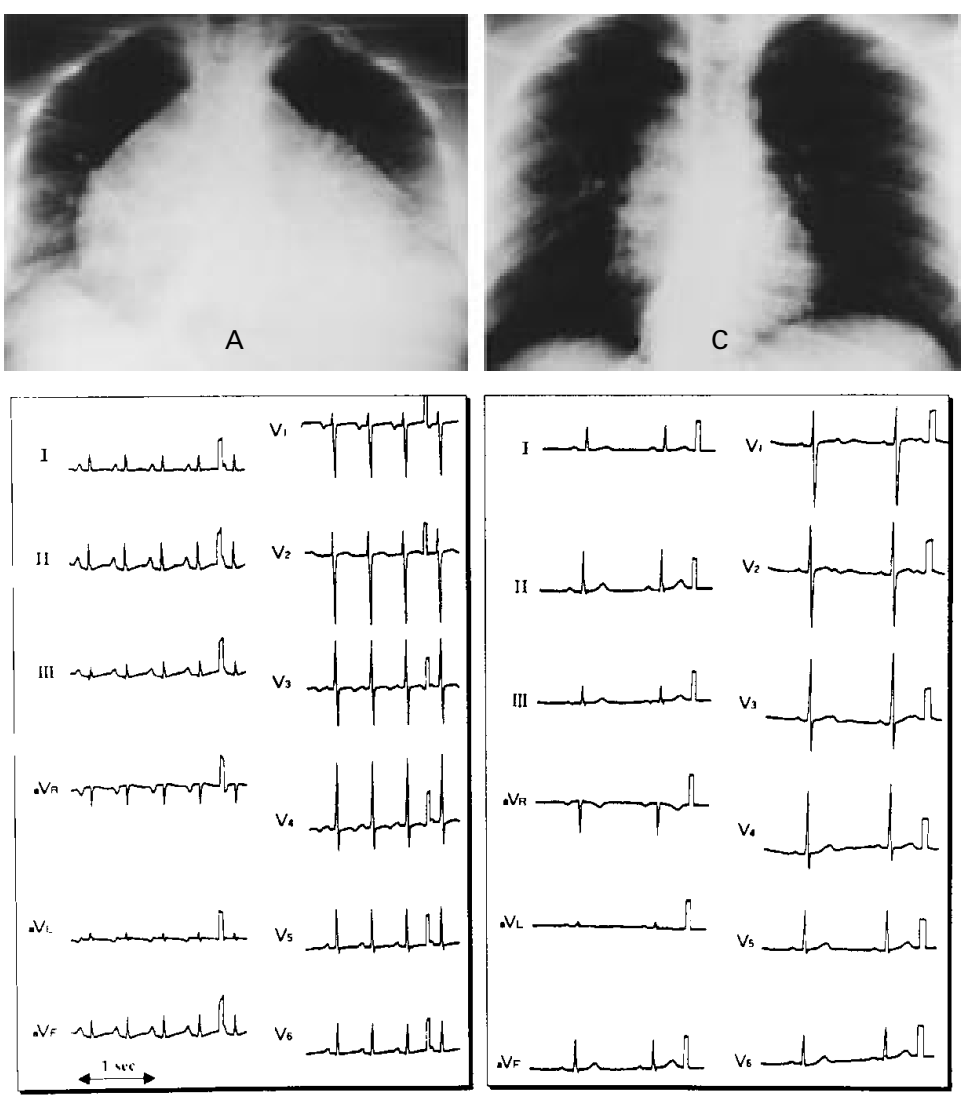

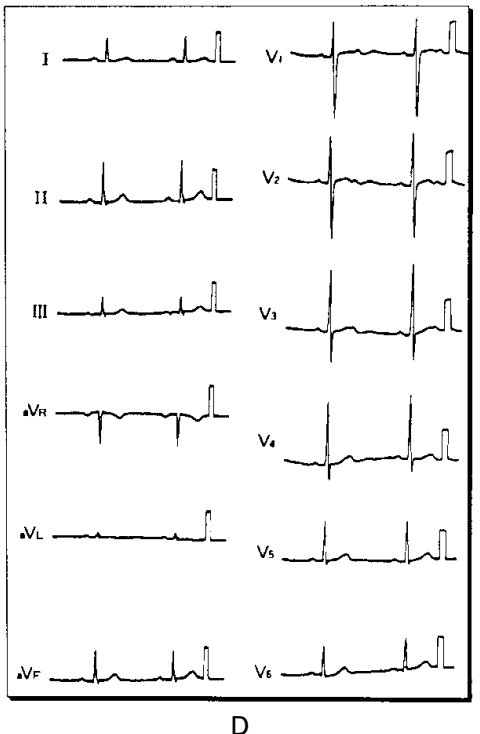

A 13 year old girl was referred with orthopnoea. Chest $x$ ray revealed pronounced cardiomegaly with a cardiothoracic ratio of $77 \%$ (panel A) and her ECG showed atrial tachycardia with a heart rate of 150 beats per minute and an upright $P$ wave configuration in the limb leads of II, III, and aVf (panel B). On echocardiography, the left ventricular ejection fraction (LVEF) was 0.21 . Digitalis, verapamil, and a small dose of metoprolol were not effective in controlling her arrhythmia. Catheter ablation was hampered by bilateral femoral vein obstruction resulting from previous surgery for a congenital genitourinary abnormality. Thereafter, we prescribed class Ic pilsicainide at an initial dose of $25 \mathrm{mg}$, and within two hours her atrial tachycardia was converted to sinus rhythm. It was maintained by a daily dose of $75 \mathrm{mg}$ pilsicainide from that day onward. After three months, the cardiothoracic ratio improved to $46 \%$ (panel C) and sinus rhythm was maintained with the same dose of pilsicainide (panel D). On echocardiography, the LVEF had improved to 0.62 . The patient has been well in the three years since, and repeated Holter recordings have indicated the maintenance of sinus rhythm.

Sustained tachycardia may cause cardiomyopathy. If catheter ablation cannot be performed as the first line treatment, antiarrhythmic drugs may be used, provided that the patient is carefully observed.

YUII NAKAZATO 